\title{
含取代噁唑结构的新型吡唑肟衍生物的合成与生物活性
}

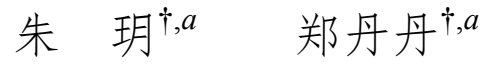 \\ 倪亚丹 ${ }^{a}$ \\ 李金峰 ${ }^{a}$ \\ 周环宇 ${ }^{a}$ \\ 胡兰萍*, $a$ \\ 李 玲 ${ }^{a}$ \\ 鞠剑峰 $*, a$ \\ 陈家正 ${ }^{a}$ 李 宏 $b$ \\ 石玉军 ${ }^{a}$ \\ 戴 红*,a,b \\ ( ${ }^{a}$ 南通大学化学化工学院 南通 226019) \\ ( ${ }^{b}$ 南通丰田化工有限公司 南通 226005)
}

\begin{abstract}
摘要 为了发现具有良好生物活性的吡唑肜化合物, 以唑螨酯为先导化合物, 在吡唑肜中引入取代腎夾结构, 设计并 制备了 20 个未见文献报道的新型吡唑肜衍生物, 利用 ${ }^{1} \mathrm{H} \mathrm{NMR},{ }^{13} \mathrm{C}$ NMR 和元素分析确证了目标产物的结构. 生物活性 测试结果显示, 部分目标化合物在 500 和 $100 \mu \mathrm{g} / \mathrm{mL}$ 浓度下对粘虫或蚜虫表现出优良的杀虫活性, 其中 5-(3-氟苯氧 基)-1,3-二甲基- $1 H$-吡唑-4-甲酰基- $O$ - $\{$ [5-(4-氯苯基)噁唑-2-基]甲基 $\}$ 肜(9j)、5-(4-氟苯氧基)-1,3-二甲基- $1 H$-吡唑-4-甲酰 基- $O$-\{[5-(4-氯苯基)噁唑-2-基]甲基 $\}$ 肜 $(9 \mathbf{k}) 、 5$-(4-叔丁基苯氧基)-1,3-二甲基- $1 H$-吡唑-4-甲酰基- $O$ - $\{[5$-(4-氯苯基)噁唑-2基]甲基\}肜(9r)和 5-(4-甲氧基苯氧基)-1,3-二甲基- $1 H$-吡唑-4-甲酰基- $O$-\{[5-(4-氯苯基)噁夾-2-基]甲基\}肜(9s)在浓度为 $100 \mu \mathrm{g} / \mathrm{mL}$ 时对粘虫的防治效果均达 100\%, 5-(4-溴苯氧基)-1,3-二甲基- $1 H$-吡唑-4-甲酰基- $O$-\{[5-(4-氟苯基)噁唑-2-基]甲 基 3 肜(9g)和 9s 在浓度为 $100 \mu \mathrm{g} / \mathrm{mL}$ 时对䖮虫的杀灭活性均为 $100 \%$. 此外, 化合物 9s 在 $500 \mu \mathrm{g} / \mathrm{mL}$ 时对朱砂叶螨的防 治效果为 $70 \%$.
\end{abstract}

关键词＼cjkstart噁唑; 吡唑肜; 合成; 生物活性

\section{Synthesis and Biological Activities of Novel Pyrazole Oxime Derivatives Bearing an Oxazole Moiety}

\author{
Zhu, Yue ${ }^{\dagger, a} \quad$ Zheng, Dandan ${ }^{\dagger, a} \quad \mathrm{Ni}_{\text {, Yadan }}{ }^{a} \quad$ Li, Jinfeng $^{a} \quad$ Zhou, Huanyu $^{a}$ \\ Hu, Lanping ${ }^{*, a} \quad$ Li, Ling $^{a} \quad$ Ju, Jianfeng ${ }^{*, a} \quad$ Chen, Jiazheng $^{a} \quad$ Li, Hong ${ }^{b}$ \\ Shi, Yujun ${ }^{a}$ Dai, Hong $*, a, b$ \\ ( ${ }^{a}$ College of Chemistry and Chemical Engineering, Nantong University, Nantong, Jiangsu 226019) \\ ( ${ }^{b}$ Nantong Fengtian Chemical Industry Co. Ltd, Nantong, Jiangsu 226005)
}

\begin{abstract}
In search of novel pyrazole oximes with good biological activities, twenty pyrazole oxime compounds were prepared by introducing an oxazole unit into pyrazole oxime based on the structure of fenpyroximate. The structures of the target compounds were confirmed by ${ }^{1} \mathrm{H}$ NMR, ${ }^{13} \mathrm{C}$ NMR and elemental analysis. Preliminary bioassay displayed that some target compounds had wonderful insecticidal activities against Oriental armyworm or Aphis medicaginis at the concentrations of 500 and $100 \mu \mathrm{g} / \mathrm{mL}$. At the concentration of $100 \mu \mathrm{g} / \mathrm{mL}, 5$-(3-fluorophenoxy)-1,3-dimethyl- $1 \mathrm{H}$-pyrazole-4-formyl-O-((5-(4-chlorophenyl)oxazol-2-yl)methyl)oxime $\quad(\mathbf{9 j}), \quad 5$-(4-fluorophenoxy)-1,3-dimethyl-1H-pyrazole-4-formyl- $O$-((5-(4-chlorophenyl)oxazol-2-yl)methyl)oxime (9k), 5-(4-t-butylphenoxy)-1,3-dimethyl-1H-pyrazole-4-formyl- $O$-((5-(4-chlorophenyl)oxazol-2yl)methyl)oxime (9r) and 5-(4-methoxyphenoxy)-1,3-dimethyl-1 $H$-pyrazole-4-formyl- $O$-((5-(4-chlorophenyl)oxazol-2-yl)methyl)oxime (9s) showed 100\% mortality rate against Oriental armyworm, and 5-(4-bromophenoxy)-1,3-dimethyl-1H-pyrazole-4-formyl-O-((5-(4-fluorophenyl)oxazol-2-yl)methyl)oxime (9g) and $\mathbf{9 s}$ exhibited $100 \%$ insecticidal property against Aphis medicaginis. Additionally, compound $9 \mathrm{~s}$ possessed $70 \%$ insecticidal activity against Tetranychus cinnabarinus at $500 \mu \mathrm{g} / \mathrm{mL}$.
\end{abstract}

Keywords oxazole; pyrazole oxime; synthesis; biological activity

\footnotetext{
*Corresponding authors. E-mail: hlp@ntu.edu.cn; ju.jf@ntu.edu.cn; daihong_2015@aliyun.com

Received October 11, 2019; revised October 29, 2019; published online November 13, 2019.

Project supported by the National Natural Science Foundation of China (Nos. 21372135, 21802075), the Natural Science Foundation of Jiangsu Province (No. BK20160413), and the Science and Technology Project Fund of Nantong City (Nos. MS12017022-4, JC2018118, MS12019060).

国家自然科学基金(Nos. 21372135, 21802075)、江苏省自然科学基金(No. BK20160413)、南通市科技计划(Nos. MS12017022-4, JC2018118, MS12019060) 资助项目.

†共同第一作者(These authors contributed equally to this work).
} 
啞唑是含有一个氮原子、一个氧原子的五元芳香杂 环。噁唑结构在农业生产和医疗保健领域多用于杀

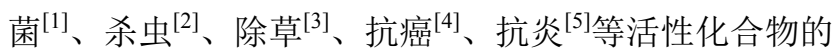
研发. 如 Xu 等开发的化合物 $\mathbf{A}$ 在浓度为 $50 \mathrm{mg} / \mathrm{L}$ 时对 粘虫的杀灭效果达 $100 \%{ }^{[6]}, \mathrm{Li}$ 课题组等开发的化合物 B 在 $200 \mathrm{mg} / \mathrm{L}$ 浓度下对粘虫具有中等水平的杀虫效果 ${ }^{[7]}$, Ye 课题组通过研究发现化合物 C 在 $20 \mathrm{mg} / \mathrm{kg}$ 浓度下对 玉米根部鲜重恢复活性达 $91.87 \%{ }^{[8]}$ (图 1). 吡唑环也是 一类重要的五元芳香杂环, 在新药创制研究中, 许多含 吡唑结构单元的药物分子构成了杀虫剂、杀菌剂、除草 剂和抗肿瘤药 ${ }^{[9 \sim 11]}$. 目前已商品化的杀虫剂有吡螨胺 (Tebufenpyrad) 和氯虫苯甲酰胺(Chlorantraniliprole) 等 (图 1) ${ }^{[12]}$. 吡唑肟作为吡唑类化合物中的重要一员, 在 农药与医药领域发挥着举足轻重的作用, 被广泛应用于

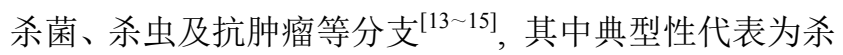
螨剂唑螨酯(Fenpyroximate, 图 1), 该化合物一直被用
来防治多种植食性螨 ${ }^{[16,17]}$. 近年来, 一些科研工作者在 唑螨酯结构基础上衍生制备出不少具有新颖结构的吡 唑肟衍生物，如 $\mathrm{Fu}$ 等研发的化合物 $\mathbf{D}$ 在浓度为 100 $\mathrm{mg} / \mathrm{L}$ 时对蚜虫和螨虫的杀灭活性分别为 $90 \%$ 和 $100 \%{ }^{[18]} ; \mathrm{He}$ 课题组研究发现化合物 $\mathbf{E}$ 在浓度为 200 $\mu \mathrm{g} / \mathrm{mL}$ 时对小菜蛾、蚜虫和螨虫均有 $100 \%$ 的防效 ${ }^{[19]}$; Shi 等合成的化合物 $\mathbf{F}$ 在浓度为 $500 \mu \mathrm{g} / \mathrm{mL}$ 时对粘虫的杀灭 活性为 $100 \%$, 同时化合物 $\mathbf{F}$ 对人肝癌细胞 HepG2 还表 现出较好的抗肿瘤活性, 其 $\mathrm{IC}_{50}$ 值为 $2.6 \mu \mathrm{mol} / \mathrm{L}$ (图 $1)^{[20]}$. 因此, 本课题组基于商品化的杀螨剂唑螨酯的结 构, 将取代的啞唑单元通过亚甲基桥引入吡唑肜化合物 中，设计制备了一系列新颖的吡唑肜类衍生物(图 2)，同 时篮选目标化合物对粘虫、蚜虫和螨虫的杀虫活性, 为 新型农用杀虫剂的研究与开发提供重要参考. 目标化合 物的合成路线见 Scheme 1.<smiles>CONC(=O)c1cc(Cl)cc(C)c1NC(=O)c1coc(-c2ccccc2Br)n1</smiles>

A<smiles>[B]c1cc(Cl)cc(C2=NC(C(C)=O)CO2)c1NC(=O)c1cc(Br)nn1-c1ncccc1Cl</smiles><smiles>Cc1ccc(-c2coc(CC3(C)OCCO3)n2)cc1</smiles><smiles>CCc1nn(C)c(C(=O)NCc2ccc(C(C)(C)C)cc2)c1Cl</smiles>

Tebufenpyrad<smiles>CNC(=O)c1cc(Cl)cc(C)c1NC(=O)c1cc(Br)nn1-c1ncccc1Cl</smiles>

Chlorantraniliprole<smiles></smiles><smiles>CC1=NN(C)c2c(C=NOc3ccc(C(=O)OC(C)(C)C)cn3)cccc2O1</smiles><smiles>CCOc1nnc(CO/N=C\c2c(C)nn(C)c2Oc2ccccc2)s1</smiles>

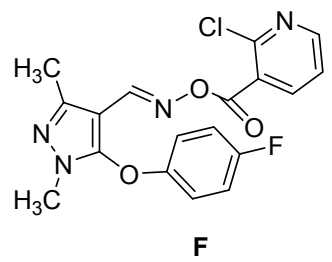

图 1 吡螨胺、氯虫苯甲酰胺、唑螨酯和化合物 $\mathbf{A} \sim \mathbf{F}$ 的化学结构

Figure 1 Chemical structures of tebufenpyrad, chlorantraniliprole, fenpyroximate, and compounds $\mathbf{A} \sim \mathbf{F}$
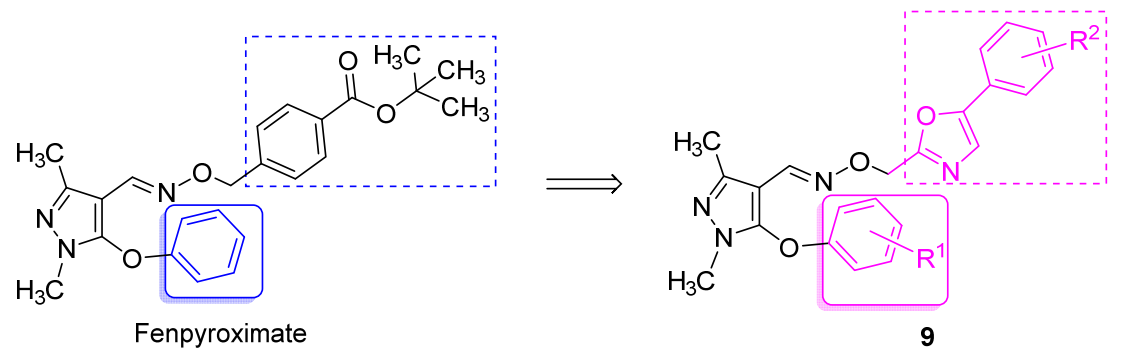

图 2 目标化合物 9 的设计

Figure 2 Design of the target compounds 9 

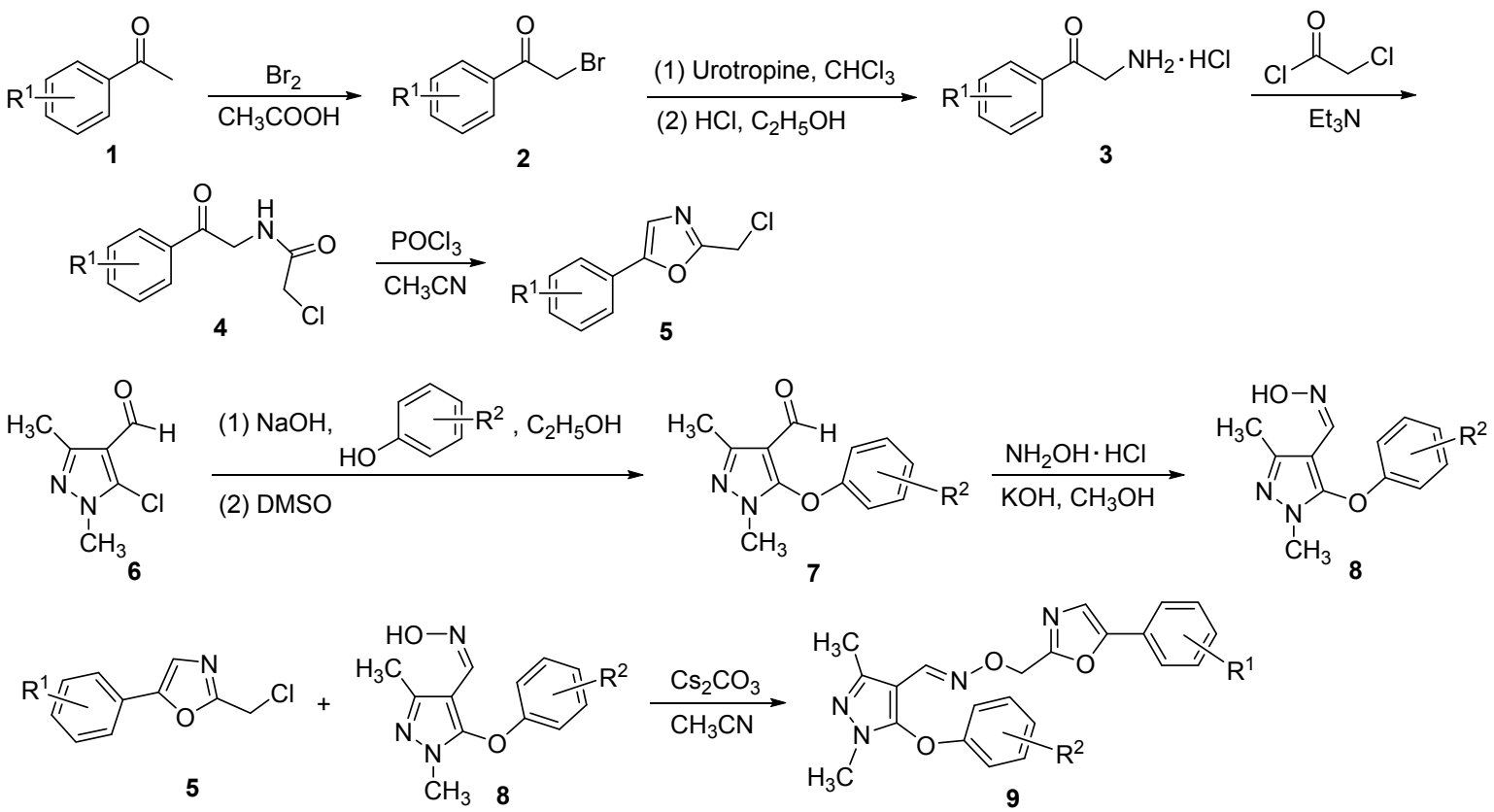

9a: $R^{1}=4-F, R^{2}=3-F ; 9 b: R^{1}=4-F, R^{2}=4-F ; 9 c: R^{1}=4-F, R^{2}=2-C l ; 9 d: R^{1}=4-F, R^{2}=3-C l ; 9 e: R^{1}=4-F, R^{2}=4-C l ; 9 f: R^{1}=4-F, R^{2}=3-$ $\mathrm{Br} ; 9 \mathrm{~g}: \mathrm{R}^{1}=4-\mathrm{F}, \mathrm{R}^{2}=4-\mathrm{Br} ; 9 \mathrm{~h}: \mathrm{R}^{1}=4-\mathrm{F}, \mathrm{R}^{2}=4-\mathrm{CH}_{3} ; 9 \mathrm{i}: \mathrm{R}^{1}=4-\mathrm{F}, \mathrm{R}^{2}=4-\mathrm{t}-\mathrm{C}_{4} \mathrm{H}_{9} ; 9 \mathrm{9j}: \mathrm{R}^{1}=4-\mathrm{Cl}, \mathrm{R}^{2}=3-\mathrm{F} ; 9 \mathbf{9 k}: \mathrm{R}^{1}=4-\mathrm{Cl}, \mathrm{R}^{2}=4-\mathrm{F} ; 9 \mathrm{9l}: \mathrm{R}^{1}=4-$ $\mathrm{Cl}, \mathrm{R}^{2}=2-\mathrm{Cl} ; 9 \mathrm{~m}: \mathrm{R}^{1}=4-\mathrm{Cl}, \mathrm{R}^{2}=3-\mathrm{Cl} ; 9 \mathrm{n}: \mathrm{R}^{1}=4-\mathrm{Cl}, \mathrm{R}^{2}=3-\mathrm{Br} ; 90: \mathrm{R}^{1}=4-\mathrm{Cl}, \mathrm{R}^{2}=4-\mathrm{Br} ; 9 \mathrm{p}: \mathrm{R}^{1}=4-\mathrm{Cl}, \mathrm{R}^{2}=\mathrm{H} ; 9 \mathrm{q}: \mathrm{R}^{1}=4-\mathrm{Cl}, \mathrm{R}^{2}=4-\mathrm{CH}_{3}$; 9r: $\mathrm{R}^{1}=4-\mathrm{Cl}, \mathrm{R}^{2}=4-t-\mathrm{C}_{4} \mathrm{H}_{9} ; 9 \mathrm{~s}: \mathrm{R}^{1}=4-\mathrm{Cl}, \mathrm{R}^{2}=4-\mathrm{OCH}_{3} ; 9 \mathrm{t}: \mathrm{R}^{1}=4-\mathrm{Cl}, \mathrm{R}^{2}=4-\mathrm{OCF}_{3}$

图式 1 目标化合物 9 的合成路线

Scheme 1 Synthetic route of target compounds 9

表 1 不同反应条件对目标化合物 9e 收率的影响

Table 1 Effect of various reaction conditions on the yield of target compound $\mathbf{9 e}$

\begin{tabular}{|c|c|c|c|c|}
\hline Entry & Base & Solvent & Reaction condition & Yield/\% \\
\hline 1 & $\mathrm{NaHCO}_{3}$ & $\mathrm{CH}_{3} \mathrm{COCH}_{3}$ & Reflux for $12 \mathrm{~h}$ & 0 \\
\hline 2 & $\mathrm{Na}_{2} \mathrm{CO}_{3}$ & $\mathrm{CH}_{3} \mathrm{COCH}_{3}$ & Reflux for $12 \mathrm{~h}$ & 0 \\
\hline 3 & $\mathrm{~K}_{2} \mathrm{CO}_{3}$ & $\mathrm{CH}_{3} \mathrm{COCH}_{3}$ & Reflux for $12 \mathrm{~h}$ & 20 \\
\hline 4 & $\mathrm{Cs}_{2} \mathrm{CO}_{3}$ & $\mathrm{CH}_{3} \mathrm{COCH}_{3}$ & Reflux for $12 \mathrm{~h}$ & 33 \\
\hline 5 & $\mathrm{NaOH}$ & $\mathrm{CH}_{3} \mathrm{COCH}_{3}$ & Reflux for $12 \mathrm{~h}$ & 0 \\
\hline 6 & $\mathrm{Na}_{2} \mathrm{CO}_{3}$ & $\mathrm{CH}_{3} \mathrm{CN}$ & Reflux for $12 \mathrm{~h}$ & 43 \\
\hline 7 & $\mathrm{~K}_{2} \mathrm{CO}_{3}$ & $\mathrm{CH}_{3} \mathrm{CN}$ & Reflux for $12 \mathrm{~h}$ & 65 \\
\hline 8 & $\mathrm{Cs}_{2} \mathrm{CO}_{3}$ & $\mathrm{CH}_{3} \mathrm{CN}$ & Reflux for $12 \mathrm{~h}$ & 76 \\
\hline 9 & $\mathrm{NaOH}$ & $\mathrm{CH}_{3} \mathrm{CN}$ & Reflux for $12 \mathrm{~h}$ & 0 \\
\hline 10 & $\mathrm{Na}_{2} \mathrm{CO}_{3}$ & DMF & $90{ }^{\circ} \mathrm{C}$ for $12 \mathrm{~h}$ & 41 \\
\hline 11 & $\mathrm{~K}_{2} \mathrm{CO}_{3}$ & DMF & $90{ }^{\circ} \mathrm{C}$ for $12 \mathrm{~h}$ & 49 \\
\hline 12 & $\mathrm{Cs}_{2} \mathrm{CO}_{3}$ & DMF & $90{ }^{\circ} \mathrm{C}$ for $12 \mathrm{~h}$ & 58 \\
\hline 13 & $\mathrm{NaOH}$ & DMF & $90{ }^{\circ} \mathrm{C}$ for $12 \mathrm{~h}$ & 0 \\
\hline
\end{tabular}

\section{1 结果与讨论}

\section{1 目标产物的合成}

在合成目标化合物 9 时, 以 $9 \mathrm{e}$ 为例, 尝试了不同的 溶剂如丙酮、乙腈和 $N, N-$ 二甲基甲酰胺(DMF), 不同的 缚酸剂如碳酸氢钠、碳酸钠、碳酸钾、碳酸铯和氢氧化 钠对化合物 9e 收率的影响(如表 1 所示). 在试验过程中 发现当选用碳酸铯为缚酸剂, 乙腈为溶剂, 加热回流反 应 $12 \mathrm{~h}$, 化合物 9e 的收率相对较高 $(76 \%)$. 所以，最终
选用该方法顺利合成出目标化合物 9.

\section{2 目标化合物的核磁共振数据解析}

以化合物 $9 \mathrm{~g}$ 为例, 对其核磁共振氢谱及碳谱数据 进行分析. 从 $9 \mathrm{~g}$ 的核磁共振氢谱数据可看出，与吡唑环 相连的 $\mathrm{CH}=\mathrm{N}$ 氢的化学位移在 $\delta 7.85$, 与噁唑环相连的 苯环上四个氢分别在 $\delta 7.59 \sim 7.62$ 和 $7.09 \sim 7.14$ 以多重 峰出现，4-溴取代的苯环上四个氢分别在 $\delta 7.37$ 和 6.75 以双重峰出现, 噁唑环 4-位上氢在 $\delta 7.24$ 以单峰出现, 与噁唑环相连的亚甲基两个氢在 $\delta 5.08$ 以单峰出现, 吡 唑环上的 1-位甲基三个氢在 $\delta 3.58$ 以单峰出现, 吡唑环 上的 3-位甲基三个氢在 $\delta 2.34$ 以单峰出现; 从 $9 \mathrm{~g}$ 的核 磁共振碳谱数据可知, 与噁唑环相连的亚甲基碳原子的 $\delta$ 值为 67.58 , 吡唑环上的 1-位甲基碳原子的 $\delta$ 值为 34.24, 吡唑环上的 3-位甲基碳原子的 $\delta$ 值为 14.59 .

\section{3 生物活性}

通过浸叶法 ${ }^{[21]}$ 对粘虫(Oriental armyworm)进行了生 物活性评价, 另外通过喷雾法 ${ }^{[22]}$ 对蚜虫(Aphis medicaginis)和朱砂叶螨(Tetranychus cinnabarinus)进行了生 物活性测定，化合物 9a 9t 的杀虫活性数据列于表 2 中. 从表 2 可看出，该系列化合物在 $500 \mu \mathrm{g} / \mathrm{mL}$ 浓度下 对粘虫均有不错的杀虫作用. 化合物 9a $\sim 9 \mathrm{t}$ 对粘虫具 有 $90 \%$ ～100\%的杀死率，与对照药唑虫酰胺的杀虫效 
表 2 目标化合物 9a 9t 的杀虫活性(死亡率 $/ \%)^{a}$

Table 2 Insecticidal activities of the target compounds $9 a \sim 9 t$ (mortality/\%)

\begin{tabular}{|c|c|c|c|c|c|c|c|c|}
\hline \multirow{2}{*}{ Compd. } & \multicolumn{3}{|c|}{ Oriental armyworm } & \multicolumn{3}{|c|}{ Aphis medicaginis } & \multicolumn{2}{|c|}{ Tetranychus cinnabarinus } \\
\hline & $500 \mu \mathrm{g} / \mathrm{mL}$ & $100 \mu \mathrm{g} / \mathrm{mL}$ & $20 \mu \mathrm{g} / \mathrm{mL}$ & $500 \mu \mathrm{g} / \mathrm{mL}$ & $100 \mu \mathrm{g} / \mathrm{mL}$ & $20 \mu \mathrm{g} / \mathrm{mL}$ & $500 \mu \mathrm{g} / \mathrm{mL}$ & $100 \mu \mathrm{g} / \mathrm{mL}$ \\
\hline 9a & 100 & 0 & - & 0 & - & - & 0 & - \\
\hline $9 b$ & 100 & 0 & - & 100 & 0 & - & 0 & - \\
\hline $9 c$ & 100 & 0 & - & 0 & - & - & 0 & - \\
\hline 9d & 90 & 0 & - & 100 & 0 & - & 0 & - \\
\hline $9 e$ & 100 & 0 & - & 100 & 20 & 0 & 0 & - \\
\hline 9f & 100 & 0 & - & 0 & - & - & 0 & - \\
\hline $9 g$ & 100 & 0 & - & 100 & 100 & 20 & 0 & - \\
\hline $9 \mathrm{~h}$ & 90 & 0 & - & 0 & - & - & 0 & - \\
\hline $9 \mathbf{i}$ & 100 & 0 & - & 0 & - & - & 0 & - \\
\hline $\mathbf{9 j}$ & 100 & 100 & 30 & 0 & - & - & 0 & - \\
\hline $9 k$ & 100 & 100 & 40 & 0 & - & - & 0 & - \\
\hline 91 & 100 & 0 & - & 0 & - & - & 0 & - \\
\hline $9 m$ & 100 & 0 & - & 0 & - & - & 0 & - \\
\hline $9 n$ & 100 & 0 & - & 0 & - & - & 0 & - \\
\hline 90 & 100 & 40 & 0 & 0 & - & - & 0 & - \\
\hline $9 p$ & 100 & 0 & - & 0 & - & - & 0 & - \\
\hline $9 q$ & 100 & 0 & - & 0 & - & - & 0 & - \\
\hline $9 \mathrm{r}$ & 100 & 100 & 0 & 0 & - & - & 0 & - \\
\hline $9 \mathrm{~s}$ & 100 & 100 & 70 & 100 & 100 & 40 & 70 & 0 \\
\hline $9 t$ & 100 & 0 & - & 0 & - & - & 0 & - \\
\hline Tolfenpyrad & 100 & 50 & 40 & - & - & - & - & - \\
\hline Imidacloprid & - & - & - & 100 & 100 & 100 & - & - \\
\hline Fenpyroximate & - & - & - & - & - & - & 100 & 100 \\
\hline
\end{tabular}

果相近. 从构效关系分析可得出, 当 $\mathrm{R}^{1}=4-\mathrm{Cl}$ 时, $\mathrm{R}^{2}$ 为 $3-\mathrm{F}(\mathbf{9 j}) 、 4-\mathrm{F}(\mathbf{9 k}) 、 4-\mathrm{Br}(\mathbf{9 o}) 、 4-t-\mathrm{C}_{4} \mathrm{H}_{9}(9 \mathbf{r}) 、 4-\mathrm{OCH}_{3}(\mathbf{9 s})$ 的化合物在浓度降到 $100 \mu \mathrm{g} / \mathrm{mL}$ 时, 对粘虫还表现出杀 虫作用，其杀灭效果分别为 $100 \%, 100 \%, 40 \%, 100 \%$ 和 $100 \%$ ，其中 $9 j 、 9 k 、 9 r$ 和 $9 \mathrm{~s}$ 对粘虫的杀虫活性明显高 于对照药唑虫酰胺的防效, 9j、9k 和 9s 在 $20 \mu \mathrm{g} / \mathrm{mL}$ 浓 度下对粘虫仍表现出 $30 \%, 40 \%$ 和 $70 \%$ 的杀灭效果. 部 分目标产物在 $500 \mu \mathrm{g} / \mathrm{mL}$ 浓度下对蚜虫呈现出优异的防 治效果, 当 $\mathrm{R}^{1}=4-\mathrm{F}$ 时, $\mathrm{R}^{2}$ 为 4-F (9b)、3-Cl (9d)、4-Cl $(9 \mathrm{e})$ 和 4-Br $(9 \mathrm{~g})$ 的化合物对蚜虫的防效均达 $100 \%$ ，与对 照药剂吡虫啉接近, 当 $\mathrm{R}^{1}=4-\mathrm{Cl}$ 时, $\mathrm{R}^{2}$ 为 $4-\mathrm{OCH}_{3}(9 \mathrm{~s})$ 的化合物对蚜虫的杀死率为 $100 \%$, 与对照药剂吡虫啉 相当; 当浓度为 $100 \mu \mathrm{g} / \mathrm{mL}$ 时, 部分化合物对蚜虫还表 现出杀虫活性, 当 $\mathrm{R}^{1}=4-\mathrm{F}$ 时, $\mathrm{R}^{2}$ 为 $4-\mathrm{Cl}(9 \mathrm{e})$ 和 $4-\mathrm{Br}(9 \mathrm{~g})$ 的化合物对蚜虫的杀死率分别为 $20 \%$ 和 $100 \%$, 其中化 合物 $9 g$ 与对照药剂吡虫啉相近, 当 $\mathrm{R}^{1}=4-\mathrm{Cl}$ 时, $\mathrm{R}^{2}$ 为 $4-\mathrm{OCH}_{3}(9 \mathrm{~s})$ 的化合物对蚜虫的防效为 $100 \%$, 与对照药 剂吡虫啉相当; 化合物 $9 g\left(R^{1}=4-F, R^{2}=4-B r\right)$ 和 $9 s\left(R^{1}\right.$ $=4-\mathrm{Cl}, \mathrm{R}^{2}=4-\mathrm{OCH}_{3}$ ) 在浓度为 $20 \mu \mathrm{g} / \mathrm{mL}$ 时对蚜虫仍表 现出 20\%和 40\%的杀虫作用, 要高于其它目标化合物的 杀虫效果. 另外, 化合物 $9 \mathrm{~s}\left(\mathrm{R}^{1}=4-\mathrm{Cl}, \mathrm{R}^{2}=4-\mathrm{OCH}_{3}\right)$ 在 浓度为 $500 \mu \mathrm{g} / \mathrm{mL}$ 时对朱砂叶螨具有 $70 \%$ 的杀灭效果，
明显优于其它化合物的杀虫作用. 通过构效关系分析也 能看出, 目标化合物 $9 \mathrm{~s}\left(\mathrm{R}^{1}=4-\mathrm{Cl}, \mathrm{R}^{2}=4-\mathrm{OCH}_{3}\right)$ 的杀虫 谱相对较广, 对所测试的三种虫都展现出了优良的杀虫 作用, 可选为先导化合物作衍生合成与生物活性研究.

\section{2 结论}

本研究以唑螨酯为先导结构, 设计合成了 20 个含 取代噁唑结构的新型吡唑肜化合物. 生物活性数据显 示, 所有化合物在 $500 \mu \mathrm{g} / \mathrm{mL}$ 浓度下对粘虫呈现出 $90 \%$ 以上的杀死率, 9j、9k、9r 和 $9 \mathrm{~s}$ 在 $100 \mu \mathrm{g} / \mathrm{mL}$ 浓度下对 粘虫具有 $100 \%$ 杀灭效果, $9 \mathrm{~s}$ 在 $20 \mu \mathrm{g} / \mathrm{mL}$ 浓度下对粘虫 仍表现出 70\%的杀虫作用; 化合物 $9 \mathrm{~b} 、 9 \mathrm{~d} 、 9 \mathrm{e} 、 9 \mathrm{~g}$ 和 9s 在 $500 \mu \mathrm{g} / \mathrm{mL}$ 时对蚜虫的杀灭效果都达 $100 \%, 9 \mathrm{~g}$ 和 9s 在 $100 \mu \mathrm{g} / \mathrm{mL}$ 下对蚜虫的杀死率仍为 $100 \%$; 另外, 9s 在 $500 \mu \mathrm{g} / \mathrm{mL}$ 时对朱砂叶螨表现出 $70 \%$ 的杀虫作用. 这 为今后继续从事吡唑肜类杀虫剂的研究提供了重要的 试验数据.

\section{3 实验部分}

\section{1 仪器与试剂}

通过 Bruker AM-400 型核磁共振波谱仪(TMS 为内 标)测定其化合物的核磁共振氢谱与核磁共振碳谱数据; 
通过 X-4 型数字显示显微熔点测定仪(温度计未经校正) 测试了化合物的熔点; 由 Yanaco-CHN CORDER MT-3 自动元素分析仪测试其化合物的元素分析; 实验用 200 300 目硅胶购于青岛海洋化工厂. 本研究中所用 试剂都为分析纯.

\section{2 化合物 1 3 和 6 8 的合成}

化合物 1 3 的合成参照文献[23]. 化合物 $6 \sim 8$ 的 合成参照文献[24]和[25].

\section{3 化合物 4 的合成}

将中间体 $3(0.05 \mathrm{~mol}) 、 50 \mathrm{~mL}$ 二氯甲烷加入 $100 \mathrm{~mL}$ 圆底烧瓶中, 搅拌下加入三乙胺 $(0.15 \mathrm{~mol})$. 冰浴条件下 向其中滴加氯乙酰氯 $(0.07 \mathrm{~mol})$, 滴加完后, 冰浴条件继 续搅拌 $2 \mathrm{~h}$, 然后室温搅拌 $8 \sim 10 \mathrm{~h}$, 薄层色谱(TLC)检测 反应进程, 完全结束后, 将混合物倒入水中, 有机相通 过盐洗、干燥后处理, 浓缩溶剂后得到粗品, 通过乙醇 重结晶后得化合物 $\mathbf{4 a}$ 和 $\mathbf{4 b}$.

2-氯- $N$-[2-(4-氟苯基)-2-氧代乙基]乙酰胺(4a): 白色 固体, 产率 58\%. m.p. $115 \sim 117{ }^{\circ} \mathrm{C} ;{ }^{1} \mathrm{H}$ NMR $(400 \mathrm{MHz}$, $\left.\mathrm{CDCl}_{3}\right) \delta: 4.14(\mathrm{~s}, 2 \mathrm{H}), 4.77(\mathrm{~d}, J=4.40 \mathrm{~Hz}, 2 \mathrm{H}), 7.18 \sim$ $7.22(\mathrm{~m}, 2 \mathrm{H}), 7.63(\mathrm{~s}, 1 \mathrm{H}), 8.01 \sim 8.05(\mathrm{~m}, 2 \mathrm{H}) ;{ }^{13} \mathrm{C} \mathrm{NMR}$ $\left(\mathrm{CDCl}_{3}, 101 \mathrm{MHz}\right) \delta: 42.47,46.37,116.21,116.43,130.67$, $130.76,165.15,166.27,167.70,191.68$. Anal. calcd for $\mathrm{C}_{10} \mathrm{H}_{9} \mathrm{ClFNO}_{2}$ : C 52.30, H 3.95, N 6.10; found C 52.13, H 3.80, N 6.26 .

2-氯- $N$-[2-(4-氯苯基)-2-氧代乙基]乙酰胺(4b): 白 色固体, 产率 65\%. m.p. $124 \sim 126{ }^{\circ} \mathrm{C} ;{ }^{1} \mathrm{H} \mathrm{NMR}\left(\mathrm{CDCl}_{3}\right.$, $400 \mathrm{MHz}) \delta: 4.14$ (s, 2H), 4.77 (d, $J=4.42 \mathrm{~Hz}, 2 \mathrm{H}), 7.50$ (d, $J=8.80 \mathrm{~Hz}, 2 \mathrm{H}), 7.62$ (s, 1H), 7.93 (d, $J=8.40 \mathrm{~Hz}$, $2 \mathrm{H}) ;{ }^{13} \mathrm{C} \mathrm{NMR}\left(\mathrm{CDCl}_{3}, 101 \mathrm{MHz}\right) \delta: 42.46,46.45,129.34$, $129.43,132.46,140.97,166.28,192.11$. Anal. calcd for $\mathrm{C}_{10} \mathrm{H}_{9} \mathrm{Cl}_{2} \mathrm{NO}_{2}$ : C 48.81, H 3.69, N 5.69; found $\mathrm{C} 48.92, \mathrm{H}$ $3.53, \mathrm{~N} 5.84$.

\section{4 化合物 5 的合成}

将中间体 4 (20 mmol)、30 mL 乙腈加入 $100 \mathrm{~mL}$ 圆 底烧瓶中, 冰浴条件下向其中滴加三氯氧磷 $(40 \mathrm{mmol})$, 滴加结束后, 升温回流反应 8 10 h, TLC 检测反应进 程, 完全结束后, 将混合物倒入水中, 用二氯甲烷萃取, 有机相通过盐洗、干燥后处理, 浓缩溶剂, 粗品经过硅 胶快速柱层析(以石油醚/乙酸乙酯为洗脱剂, $V: V=$ $8: 1)$ 分离形成化合物 $\mathbf{5 a}$ 和 $\mathbf{5 b}$.

2-氯甲基-5-(4-氟苯基)噁唑(5a): 黄色油状物, 产率 $52 \%$. ${ }^{1} \mathrm{H}$ NMR (400 MHz, $\mathrm{CDCl}_{3}$ ) $\delta: 4.68$ (s, 2H), 7.11 $7.16(\mathrm{~m}, 2 \mathrm{H}), 7.27(\mathrm{~s}, 1 \mathrm{H}), 7.62 \sim 7.66(\mathrm{~m}, 2 \mathrm{H}) ;{ }^{13} \mathrm{C} \mathrm{NMR}$ $\left(101 \mathrm{MHz}, \mathrm{DMSO}-d_{6}\right) \delta: 36.68,116.67,116.89,123.54$,
124.13, 126.98, 151.47, 159.10, 161.45, 163.91. Anal. calcd for $\mathrm{C}_{10} \mathrm{H}_{7} \mathrm{ClFNO}$ : $\mathrm{C} 56.76, \mathrm{H} 3.33, \mathrm{~N} 6.62$; found $\mathrm{C}$ $56.60, \mathrm{H} 3.48$, N 6.50 .

2-氯甲基-5-(4-氯苯基)噁唑(5b): 黄色油状物, 产率 $56 \%$. ${ }^{1} \mathrm{H}$ NMR $\left(\mathrm{CDCl}_{3}, 400 \mathrm{MHz}\right) \delta: 4.67$ (s, 2H), 7.31 (s, $1 \mathrm{H}), 7.41$ (d, $J=8.42 \mathrm{~Hz}, 2 \mathrm{H}), 7.59$ (d, $J=8.80 \mathrm{~Hz}, 2 \mathrm{H})$; ${ }^{13} \mathrm{C} \mathrm{NMR}\left(\mathrm{CDCl}_{3}, 101 \mathrm{MHz}\right) \delta: 35.95,122.80,125.67$, $125.86,129.27,134.79,151.83,158.63$. Anal. calcd for $\mathrm{C}_{10} \mathrm{H}_{7} \mathrm{Cl}_{2}-\mathrm{NO}$ : C 52.66, H 3.09, N 6.14; found C 52.83, H 3.23, N 6.01 .

\subsection{5 -取代苯氧基-1,3-二甲基-1H-吡唑-4-甲酰基- O-[(5-取代苯基噁唑-2-基)甲基]肜(9a〜 9t)的合成}

将吡唑甲酰基肟 8 (2 mmol)、化合物 5 (2.5 mmol) 及 $25 \mathrm{~mL}$ 乙腈加入一 $50 \mathrm{~mL}$ 圆底烧瓶中, 室温摚拌下, 向其中加入 $4 \mathrm{mmol}$ 碳酸铯. 加完后升温至回流, 反应 10 20 h, TLC 跟踪反应的进程. 反应完成后，趁热过 滤, 母液浓缩至干, 所得粗品通过硅胶快速柱层析[石 油醚/乙酸乙酯 $(V: V=4: 1)$ 为洗脱剂]分离得到目标产 物 9a $\sim 9$ t.

5-(3-氟苯氧基)-1,3-二甲基- $1 H$-吡唑-4-甲酰基- $O$ $\{[5-(4$-氟苯基)噁唑-2-基]甲基 $\}$ 肜(9a): 黄色固体，产率 62\%. m.p. 63 $65{ }^{\circ} \mathrm{C}$; ${ }^{1} \mathrm{H}$ NMR (400 MHz, $\left.\mathrm{CDCl}_{3}\right) \delta$ : $2.28(\mathrm{~s}, 3 \mathrm{H}), 3.52(\mathrm{~s}, 3 \mathrm{H}), 5.02(\mathrm{~s}, 2 \mathrm{H}), 6.53 \sim 6.72(\mathrm{~m}$, $3 \mathrm{H}), 7.02 \sim 7.06(\mathrm{~m}, 2 \mathrm{H}), 7.12 \sim 7.17(\mathrm{~m}, 2 \mathrm{H}), 7.51 \sim 7.55$ $(\mathrm{m}, 2 \mathrm{H}), 7.79(\mathrm{~s}, 1 \mathrm{H}) ;{ }^{13} \mathrm{C} \mathrm{NMR}\left(101 \mathrm{MHz}, \mathrm{CDCl}_{3}\right) \delta$ : $14.69,34.26,67.61,99.93,103.45,110.88,115.94,116.16$, $121.80,124.18,126.18,126.26,130.92,141.61,147.24$, $151.23,157.65,160.02,161.50,162.25,163.97,164.71$. Anal. calcd for $\mathrm{C}_{22} \mathrm{H}_{18} \mathrm{~F}_{2} \mathrm{~N}_{4} \mathrm{O}_{3}$ : C 62.26, $\mathrm{H}$ 4.28, N 13.20; found $\mathrm{C} 62.13, \mathrm{H} 4.43, \mathrm{~N} 13.37$.

5-(4-氟苯氧基)-1,3-二甲基- $1 H$-吡唑-4-甲酰基$O$ - $\{[$-(4-氟苯基)啞唑-2-基]甲基 $\}$ 肜(9b)：黄色固体，产 率 65\%. m.p. $73 \sim 75{ }^{\circ} \mathrm{C} ;{ }^{1} \mathrm{H}$ NMR (400 $\left.\mathrm{MHz} \mathrm{CDCl}_{3}\right) \delta$ : $2.34(\mathrm{~s}, 3 \mathrm{H}), 3.60(\mathrm{~s}, 3 \mathrm{H}), 5.09(\mathrm{~s}, 2 \mathrm{H}), 6.82 \sim 6.85(\mathrm{~m}$, $2 \mathrm{H}), 6.94 \sim 6.98(\mathrm{~m}, 2 \mathrm{H}), 7.09 \sim 7.13(\mathrm{~m}, 2 \mathrm{H}), 7.24(\mathrm{~s}$, $1 \mathrm{H}), 7.58 \sim 7.62(\mathrm{~m}, 2 \mathrm{H}), 7.84(\mathrm{~s}, 1 \mathrm{H}) ;{ }^{13} \mathrm{C}$ NMR $(101$ $\left.\mathrm{MHz}, \mathrm{CDCl}_{3}\right) \delta: 14.65,34.21,67.57,99.60,115.94$, $116.16,116.62,121.75,124.14,126.18,141.68,147.19$, $148.05,151.20,152.53,157.56,160.04,161.49,163.97$. Anal. calcd for $\mathrm{C}_{22} \mathrm{H}_{18} \mathrm{~F}_{2} \mathrm{~N}_{4} \mathrm{O}_{3}$ : C 62.26, $\mathrm{H}$ 4.28, N 13.20; found $\mathrm{C} 62.43, \mathrm{H} 4.15, \mathrm{~N} 13.06$.

5-(2-氯苯氧基)-1,3-二甲基- $1 H$-吡唑-4-甲酰基$O$-\{[5-(4-氟苯基)噁唑-2-基]甲基 $\}$ 肜(9c)：黄色油状物， 产率 63\%. ${ }^{1} \mathrm{H}$ NMR $\left(400 \mathrm{MHz}, \mathrm{CDCl}_{3}\right) \delta: 2.35(\mathrm{~s}, 3 \mathrm{H})$, $3.64(\mathrm{~s}, 3 \mathrm{H}), 5.08(\mathrm{~s}, 2 \mathrm{H}), 6.67 \sim 6.92(\mathrm{~m}, 1 \mathrm{H}), 7.00 \sim 7.13$ 
(m, 4H), $7.24(\mathrm{~s}, 1 \mathrm{H}), 7.41 \sim 7.43(\mathrm{~m}, 1 \mathrm{H}), 7.59 \sim 7.62(\mathrm{~m}$, 2H), $7.82(\mathrm{~s}, 1 \mathrm{H}) ;{ }^{13} \mathrm{C} \mathrm{NMR}\left(101 \mathrm{MHz}, \mathrm{CDCl}_{3}\right) \delta: 14.65$, $34.24,67.58,99.69,115.94,116.16,121.74,122.82$, $124.14,124.72,126.18,126.26,128.01,130.97,141.46$, $147.18,147.59,151.20,152.04,160.01,161.48$. Anal. calcd for $\mathrm{C}_{22} \mathrm{H}_{18} \mathrm{ClFN}_{4} \mathrm{O}_{3}$ : C 59.94, H 4.12, N 12.71; found C 59.78, H 4.25, N 12.54 .

5-(3-氯苯氧基)-1,3-二甲基- $1 H$-吡唑-4-甲酰基- $O$ \{[5-(4-氟苯基)啞唑-2-基]甲基\}肟(9d): 黄色油状物, 产 率 69\%. ${ }^{1} \mathrm{H}$ NMR (400 MHz, $\mathrm{CDCl}_{3}$ ) $\delta: 2.38$ (s, 3H), 3.63 $(\mathrm{s}, 3 \mathrm{H}), 5.10(\mathrm{~s}, 2 \mathrm{H}), 6.75 \sim 6.78(\mathrm{~m}, 1 \mathrm{H}), 6.90(\mathrm{~s}, 1 \mathrm{H})$, $7.04 \sim 7.23(\mathrm{~m}, 4 \mathrm{H}), 7.25(\mathrm{~s}, 1 \mathrm{H}), 7.59 \sim 7.62(\mathrm{~m}, 2 \mathrm{H})$, $7.85(\mathrm{~s}, 1 \mathrm{H}) ;{ }^{13} \mathrm{C}$ NMR $\left(101 \mathrm{MHz}, \mathrm{CDCl}_{3}\right) \delta: 14.60,34.28$, 67.62, 99.98, 113.56, 115.94, 116.16, 121.77, 124.09, $126.18,126.26,130.78,135.48,141.48,147.21,151.22$, $157.04,159.97,161.50,163.97$. Anal. calcd for $\mathrm{C}_{22} \mathrm{H}_{18} \mathrm{Cl}-$ $\mathrm{FN}_{4} \mathrm{O}_{3}$ : C 59.94, $\mathrm{H} 4.12, \mathrm{~N} \mathrm{12.71;} \mathrm{found} \mathrm{C} 60.12, \mathrm{H} 4.01$, N 12.86 .

5-(4-氯苯氧基)-1,3-二甲基- $1 H$-吡唑-4-甲酰基- $O$ \{[5-(4-氟苯基)啞唑-2-基]甲基 $\}$ 肜(9e)：黄色固体，产率 76\%. m.p. 91 93 ${ }^{\circ} \mathrm{C}$; ${ }^{1} \mathrm{H}$ NMR (400 MHz, $\mathrm{CDCl}_{3}$ ) $\delta$ : 2.34 (s, 3H), 3.59 (s, 3H), $5.09(\mathrm{~s}, 2 \mathrm{H}), 6.81$ (d, $J=9.22$ $\mathrm{Hz}, 2 \mathrm{H}), 7.09 \sim 7.13(\mathrm{~m}, 2 \mathrm{H}), 7.21 \sim 7.24(\mathrm{~m}, 3 \mathrm{H}), 7.59 \sim$ $7.62(\mathrm{~m}, 2 \mathrm{H}), 7.85(\mathrm{~s}, 1 \mathrm{H}) ;{ }^{13} \mathrm{C} \mathrm{NMR}\left(101 \mathrm{MHz}, \mathrm{CDCl}_{3}\right) \delta$ : 14.60, 34.24, 67.59, 99.81, 115.96, 116.18, 116.64, 121.76, $124.16,126.19,128.84,129.92,141.60,147.25,147.52$, $151.20,155.12,160.03,161.50,163.98$. Anal. calcd for

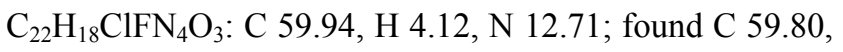
$\mathrm{H} 4.03, \mathrm{~N} 12.80$.

5-(3-溴苯氧基)-1,3-二甲基- $1 H$-吡唑-4-甲酰基$O$-\{[5-(4-氟苯基)噁唑-2-基]甲基 $\}$ 肜(9f): 黄色油状物, 产率 70\%. ${ }^{1} \mathrm{H}$ NMR (400 MHz, $\left.\mathrm{CDCl}_{3}\right) \delta: 2.35(\mathrm{~s}, 3 \mathrm{H})$, $3.59(\mathrm{~s}, 3 \mathrm{H}), 5.09(\mathrm{~s}, 2 \mathrm{H}), 6.79 \sim 7.20(\mathrm{~m}, 6 \mathrm{H}), 7.25(\mathrm{~s}$, $1 \mathrm{H}), 7.59 \sim 7.62(\mathrm{~m}, 2 \mathrm{H}), 7.86(\mathrm{~s}, 1 \mathrm{H}) ;{ }^{13} \mathrm{C}$ NMR $(101$ $\left.\mathrm{MHz}, \mathrm{CDCl}_{3}\right) \delta: 14.62,34.27,67.59$, 99.92, 114.00, $115.93,116.15,118.80,121.77,123.18,124.15,126.18$, $126.26,126.95,131.04,141.54,147.27,151.20,157.08$, 160.00, 161.48, 163.95. Anal. calcd for $\mathrm{C}_{22} \mathrm{H}_{18} \mathrm{BrFN}_{4} \mathrm{O}_{3}: \mathrm{C}$ 54.45, H 3.74, N 11.54; found C 54.28, H 3.90, N 11.65.

5-(4-溴苯氧基)-1,3-二甲基- $1 H$-吡唑-4-甲酰基 $O$ - $\{[5$-(4-氟苯基)噁唑-2-基]甲基 $\}$ 肜(9g)：黄色固体，产 率 73\%. m.p. 89 91 ${ }^{\circ} \mathrm{C} ;{ }^{1} \mathrm{H}$ NMR $\left(400 \mathrm{MHz}, \mathrm{CDCl}_{3}\right) \delta$ : $2.34(\mathrm{~s}, 3 \mathrm{H}), 3.58(\mathrm{~s}, 3 \mathrm{H}), 5.08(\mathrm{~s}, 2 \mathrm{H}), 6.75(\mathrm{~d}, J=9.20$ $\mathrm{Hz}, 2 \mathrm{H}), 7.09 \sim 7.14(\mathrm{~m}, 2 \mathrm{H}), 7.24(\mathrm{~s}, 1 \mathrm{H}), 7.37(\mathrm{~d}, J=$ $8.82 \mathrm{~Hz}, 2 \mathrm{H}), 7.59 \sim 7.62(\mathrm{~m}, 2 \mathrm{H}), 7.85(\mathrm{~s}, 1 \mathrm{H}) ;{ }^{13} \mathrm{C} \mathrm{NMR}$ $\left(101 \mathrm{MHz}, \mathrm{CDCl}_{3}\right) \delta: 14.59,34.24,67.58,99.81,115.94$, $116.16,117.06,121.78,124.15,126.17,126.26,132.85$, $141.57,147.24,147.38,151.17,155.67,160.01,161.48$, 163.96. Anal. calcd for $\mathrm{C}_{22} \mathrm{H}_{18} \mathrm{BrFN}_{4} \mathrm{O}_{3}$ : C 54.45, H 3.74, N 11.54; found C 54.61, H 3.58, N 11.39.

5-(4-甲基苯氧基)-1,3-二甲基- $1 H$-吡唑-4-甲酰基$O$-\{[5-(4-氟苯基)噁唑-2-基]甲基 $\}$ 肜(9h)：黄色油状物， 产率 80\%. ${ }^{1} \mathrm{H} \mathrm{NMR}\left(400 \mathrm{MHz}, \mathrm{CDCl}_{3}\right) \delta: 2.29(\mathrm{~s}, 3 \mathrm{H})$, $2.36(\mathrm{~s}, 3 \mathrm{H}), 3.60(\mathrm{~s}, 3 \mathrm{H}), 5.11(\mathrm{~s}, 2 \mathrm{H}), 6.76(\mathrm{~d}, J=8.80$ $\mathrm{Hz}, 2 \mathrm{H}), 7.06 \sim 7.13(\mathrm{~m}, 4 \mathrm{H}), 7.25(\mathrm{~s}, 1 \mathrm{H}), 7.59 \sim 7.62(\mathrm{~m}$, 2H), $7.82(\mathrm{~s}, 1 \mathrm{H}) ;{ }^{13} \mathrm{C}$ NMR (101 MHz, $\left.\mathrm{CDCl}_{3}\right) \delta: 14.89$, 20.57, 34.18, 67.54, 99.69, 115.19, 115.93, 116.15, 121.70, $124.15,126.18,126.26,130.43,132.65,133.39,141.94$, 147.00, 148.59, 151.24, 154.58, 160.13, 161.49, 163.97. Anal. calcd for $\mathrm{C}_{23} \mathrm{H}_{21} \mathrm{FN}_{4} \mathrm{O}_{3}: \mathrm{C}$ 65.71, $\mathrm{H}$ 5.03, N 13.33; found C 65.53, H 5.19, N 13.20.

5-(4-叔丁基苯氧基)-1,3-二甲基- $1 H$-吡唑-4-甲酰基$O$-\{[5-(4-氟苯基)噁唑-2-基]甲基 $\}$ 肜(9i)：黄色油状物， 产率 70\%. ${ }^{1} \mathrm{H}$ NMR (400 $\left.\mathrm{MHz}, \mathrm{CDCl}_{3}\right) \delta: 1.29$ (s, 9H), $2.37(\mathrm{~s}, 3 \mathrm{H}), 3.60(\mathrm{~s}, 3 \mathrm{H}), 5.11(\mathrm{~s}, 2 \mathrm{H}), 6.79$ (d, $J=8.82$ $\mathrm{Hz}, 2 \mathrm{H}), 7.09 \sim 7.13(\mathrm{~m}, 2 \mathrm{H}), 7.26(\mathrm{~s}, 1 \mathrm{H}), 7.30$ (d, $J=$ $8.80 \mathrm{~Hz}, 2 \mathrm{H}), 7.59 \sim 7.63(\mathrm{~m}, 2 \mathrm{H}), 7.84(\mathrm{~s}, 1 \mathrm{H}) ;{ }^{13} \mathrm{C} \mathrm{NMR}$ $\left(101 \mathrm{MHz}, \mathrm{CDCl}_{3}\right) \delta: 15.02,31.40,34.21,34.30,67.55$, $99.74,114.75,115.94,116.16,121.75,124.15,126.19$, $126.27,126.83,132.66,142.00,146.72,146.98,148.58$, $151.26,154.48,160.09,161.49,163.97$. Anal. calcd for $\mathrm{C}_{26} \mathrm{H}_{27} \mathrm{FN}_{4} \mathrm{O}_{3}$ : C 67.52, H 5.88, N 12.11; found $\mathrm{C} 67.68, \mathrm{H}$ $5.73, \mathrm{~N} 12.07$.

5-(3-氟苯氧基)-1,3-二甲基- $1 H$-吡唑-4-甲酰基- $O$ $\{[5-(4$-氯苯基)噁唑-2-基]甲基 $\}$ 肜 $(9 \mathrm{j})$ : 黄色固体, 产率 67\%. m.p. 95 97 ${ }^{\circ} \mathrm{C}$; ${ }^{1} \mathrm{H}$ NMR (400 MHz, $\left.\mathrm{CDCl}_{3}\right) \delta$ : $2.35(\mathrm{~s}, 3 \mathrm{H}), 3.60(\mathrm{~s}, 3 \mathrm{H}), 5.09(\mathrm{~s}, 2 \mathrm{H}), 6.60 \sim 6.69(\mathrm{~m}$, $3 \mathrm{H}), 7.20 \sim 7.25(\mathrm{~m}, 1 \mathrm{H}), 7.29(\mathrm{~s}, 1 \mathrm{H}), 7.38(\mathrm{~d}, J=8.80$ $\mathrm{Hz}, 2 \mathrm{H}), 7.55$ (d, $J=8.82 \mathrm{~Hz}, 2 \mathrm{H}), 7.86(\mathrm{~s}, 1 \mathrm{H}) ;{ }^{13} \mathrm{C} \mathrm{NMR}$ $\left(101 \mathrm{MHz} \mathrm{CDCl}_{3}\right) \delta: 14.66,34.25,67.58,99.90,103.43$, $110.86,122.50,125.53,126.29,129.15,130.81,134.33$, $141.63,147.22,151.02,157.51,160.27,162.22,164.69$. Anal. calcd for $\mathrm{C}_{22} \mathrm{H}_{18} \mathrm{ClFN}_{4} \mathrm{O}_{3}$ : C 59.94, $\mathrm{H} 4.12, \mathrm{~N} 12.71$; found $\mathrm{C} 60.06, \mathrm{H} 4.28, \mathrm{~N} 12.88$.

5-(4-氟苯氧基)-1,3-二甲基- $1 H$-吡唑-4-甲酰基$O$-\{[5-(4-氯苯基)噁唑-2-基]甲基 $\}$ 肜(9k)：黄色固体，产 率 72\%. m.p. 101 103 ${ }^{\circ} \mathrm{C} ;{ }^{1} \mathrm{H} \mathrm{NMR}\left(400 \mathrm{MHz}, \mathrm{CDCl}_{3}\right.$ ) $\delta: 2.27(\mathrm{~s}, 3 \mathrm{H}), 3.53(\mathrm{~s}, 3 \mathrm{H}), 5.02(\mathrm{~s}, 2 \mathrm{H}), 6.74 \sim 6.90(\mathrm{~m}$, 4H), $7.21(\mathrm{~s}, 1 \mathrm{H}), 7.31(\mathrm{~d}, J=8.42 \mathrm{~Hz}, 2 \mathrm{H}), 7.48$ (d, $J=$ $8.82 \mathrm{~Hz}, 2 \mathrm{H}), 7.76(\mathrm{~s}, 1 \mathrm{H}) ;{ }^{13} \mathrm{C} \mathrm{NMR}\left(101 \mathrm{MHz}, \mathrm{CDCl}_{3}\right) \delta$ : 
$14.66,34.24,67.57,99.59,116.34,116.63,122.50,125.54$, $126.29,129.18,134.27,141.74,147.21,148.06,151.03$, 152.54, 157.57, 159.98, 160.32. Anal. calcd for $\mathrm{C}_{22} \mathrm{H}_{18} \mathrm{ClFN}_{4} \mathrm{O}_{3}$ : C 59.94, $\mathrm{H} 4.12, \mathrm{~N} 12.71$; found C 59.76, $\mathrm{H} 4.03, \mathrm{~N} 12.79$.

5-(2-氯苯氧基)-1,3-二甲基- $1 H$-吡唑-4-甲酰基$O$-\{[5-(4-氯苯基)啞唑-2-基]甲基 $\}$ 肜(91): 黄色固体, 产 率 65\%. m.p. 70 72 ${ }^{\circ} \mathrm{C} ;{ }^{1} \mathrm{H}$ NMR $\left(400 \mathrm{MHz}, \mathrm{CDCl}_{3}\right) \delta$ : $2.27(\mathrm{~s}, 3 \mathrm{H}), 3.56(\mathrm{~s}, 3 \mathrm{H}), 5.01(\mathrm{~s}, 2 \mathrm{H}), 6.59 \sim 6.61(\mathrm{~m}$, $1 \mathrm{H}), 6.92 \sim 7.07(\mathrm{~m}, 2 \mathrm{H}), 7.21(\mathrm{~s}, 1 \mathrm{H}), 7.30 \sim 7.35(\mathrm{~m}$, $3 \mathrm{H}), 7.48(\mathrm{~d}, J=8.82 \mathrm{~Hz}, 2 \mathrm{H}), 7.75(\mathrm{~s}, 1 \mathrm{H}) ;{ }^{13} \mathrm{C}$ NMR $(101$ $\left.\mathrm{MHz}, \mathrm{CDCl}_{3}\right) \delta: 14.66,34.25,67.58,99.68,115.67$, $122.49,122.82,124.70,125.55,126.30,128.01,129.17$, $130.98,134.36,141.54,147.21,147.58,151.02,152.07$, 160.29. Anal. calcd for $\mathrm{C}_{22} \mathrm{H}_{18} \mathrm{Cl}_{2} \mathrm{~N}_{4} \mathrm{O}_{3}$ : C 57.78, H 3.97, $\mathrm{N}$ 12.25; found C 57.95, H 3.83, N 12.09 .

5-(3-氯苯氧基)-1,3-二甲基- $1 H$-吡唑-4-甲酰基$O$ - $\{$ [5-(4-氯苯基)噁唑-2-基]甲基 $\}$ 肟 $(9 \mathrm{~m})$ : 黄色固体, 产 率 70\%. m.p. 92 94 ${ }^{\circ} \mathrm{C} ;{ }^{1} \mathrm{H}$ NMR $\left(400 \mathrm{MHz}, \mathrm{CDCl}_{3}\right) \delta$ : $2.28(\mathrm{~s}, 3 \mathrm{H}), 3.53(\mathrm{~s}, 3 \mathrm{H}), 5.02(\mathrm{~s}, 2 \mathrm{H}), 6.67 \sim 6.98(\mathrm{~m}$, $3 \mathrm{H}), 7.10 \sim 7.14(\mathrm{~m}, 1 \mathrm{H}), 7.22(\mathrm{~s}, 1 \mathrm{H}), 7.32(\mathrm{~d}, J=8.82$ $\mathrm{Hz}, 2 \mathrm{H}), 7.49$ (d, $J=8.80 \mathrm{~Hz}, 2 \mathrm{H}), 7.79$ (s, 1H); ${ }^{13} \mathrm{C} \mathrm{NMR}$ $\left(101 \mathrm{MHz}, \mathrm{CDCl}_{3}\right) \delta: 13.58,33.25,66.57,98.90,112.51$, $114.94,121.47,123.03,124.51,125.26,128.14,129.72$, 133.32, 134.44, 140.53, 146.21, 150.00, 156.03, 159.22. Anal. calcd for $\mathrm{C}_{22} \mathrm{H}_{18} \mathrm{Cl}_{2} \mathrm{~N}_{4} \mathrm{O}_{3}$ : C 57.78, H 3.97, N 12.25; found $\mathrm{C}$ 57.63, $\mathrm{H} 4.11, \mathrm{~N} 12.42$.

5-(3-溴苯氧基)-1,3-二甲基- $1 H$-吡唑-4-甲酰基$O$-\{[5-(4-氯苯基)噁唑-2-基]甲基 $\}$ 肜(9n): 黄色固体, 产 率 73\%. m.p. 100 102 ${ }^{\circ} \mathrm{C} ;{ }^{1} \mathrm{H}$ NMR (400 MHz, $\mathrm{CDCl}_{3}$ ) $\delta: 2.36(\mathrm{~s}, 3 \mathrm{H}), 3.60(\mathrm{~s}, 3 \mathrm{H}), 5.09(\mathrm{~s}, 2 \mathrm{H}), 6.79 \sim 6.81(\mathrm{~m}$, $1 \mathrm{H}), 7.04 \sim 7.20(\mathrm{~m}, 3 \mathrm{H}), 7.30(\mathrm{~s}, 1 \mathrm{H}), 7.39(\mathrm{~d}, J=8.42$ $\mathrm{Hz}, 2 \mathrm{H}), 7.50$ (d, $J=8.80 \mathrm{~Hz}, 2 \mathrm{H}), 7.86(\mathrm{~s}, 1 \mathrm{H}) ;{ }^{13} \mathrm{C}$ NMR $\left(101 \mathrm{MHz}, \mathrm{CDCl}_{3}\right) \delta: 14.57,34.28,67.58,99.93,114.01$, $118.81,122.48,123.18,125.54,126.27,126.98,129.16$, $131.04,134.34,141.52,147.20,147.23,151.02,157.03$, 160.24. Anal. calcd for $\mathrm{C}_{22} \mathrm{H}_{18} \mathrm{BrClN}_{4} \mathrm{O}_{3}$ : C 52.66, H 3.62, N 11.17; found C 52.50, H 3.49, N 11.32.

5-(4-溴苯氧基)-1,3-二甲基- $1 H$-吡唑-4-甲酰基$O$-\{[5-(4-氯苯基)噁唑-2-基]甲基 $\}$ 肜(9o): 黄色固体, 产 率 78\%. m.p. 96 98 ${ }^{\circ} \mathrm{C} ;{ }^{1} \mathrm{H}$ NMR $\left(400 \mathrm{MHz}, \mathrm{CDCl}_{3}\right) \delta$ : $2.34(\mathrm{~s}, 3 \mathrm{H}), 3.58(\mathrm{~s}, 3 \mathrm{H}), 5.08(\mathrm{~s}, 2 \mathrm{H}), 6.75(\mathrm{~d}, J=9.20$ $\mathrm{Hz}, 2 \mathrm{H}), 7.29(\mathrm{~s}, 1 \mathrm{H}), 7.35 \sim 7.40(\mathrm{~m}, 4 \mathrm{H}), 7.56(\mathrm{~d}, J=$ $8.80 \mathrm{~Hz}, 2 \mathrm{H}), 7.85(\mathrm{~s}, 1 \mathrm{H}) ;{ }^{13} \mathrm{C} \mathrm{NMR}\left(101 \mathrm{MHz}, \mathrm{CDCl}_{3}\right) \delta$ : $14.57,34.24,67.56,99.79,116.21,117.05,122.50,125.53$,
$126.28,129.16,132.85,134.34,141.61,147.24,147.37$, 150.99, 155.66, 160.27. Anal. calcd for $\mathrm{C}_{22} \mathrm{H}_{18} \mathrm{BrClN}_{4} \mathrm{O}_{3}$ : C 52.66, H 3.62, N 11.17; found C 52.56, H 3.78, N 11.02.

5-苯氧基-1,3-二甲基- $1 H$-吡唑-4-甲酰基- $O$ - $\{[5$-(4氯苯基)啞唑-2-基]甲基\}肟(9p)：黄色油状物，产率 75\%. ${ }^{1} \mathrm{H}$ NMR (400 MHz, $\mathrm{CDCl}_{3}$ ) $\delta: 2.29$ (s, 3H), 3.52 (s, 3H), $5.02(\mathrm{~s}, 2 \mathrm{H}), 6.80(\mathrm{~d}, J=8.02 \mathrm{~Hz}, 2 \mathrm{H}), 6.98 \sim 7.23(\mathrm{~m}$, 4H), 7.31 (d, $J=8.40 \mathrm{~Hz}, 2 \mathrm{H}), 7.48$ (d, $J=8.80 \mathrm{~Hz}, 2 \mathrm{H})$, $7.77(\mathrm{~s}, 1 \mathrm{H}) ;{ }^{13} \mathrm{C}$ NMR $\left(101 \mathrm{MHz}, \mathrm{CDCl}_{3}\right) \delta: 14.88,34.24$, 67.56, 99.78, 115.34, 122.50, 123.80, 125.56, 126.32, $129.17,130.02,134.35,141.95,147.09,148.17,151.05$, 156.65, 160.37. Anal. calcd for $\mathrm{C}_{22} \mathrm{H}_{19} \mathrm{ClN}_{4} \mathrm{O}_{3}$ : C 62.49, $\mathrm{H}$ 4.53, N 13.25; found C 62.60, H 4.38, N 13.07.

5-(4-甲基苯氧基)-1,3-二甲基- $1 H$-吡唑-4-甲酰基$O$-\{[5-(4-氯苯基)噁唑-2-基]甲基 $\}$ 肜(9q)：黄色固体，产 率 80\%. m.p. 63 $65{ }^{\circ} \mathrm{C} ;{ }^{1} \mathrm{H}$ NMR $\left(400 \mathrm{MHz}, \mathrm{CDCl}_{3}\right) \delta$ : $2.21(\mathrm{~s}, 3 \mathrm{H}), 2.28(\mathrm{~s}, 3 \mathrm{H}), 3.51(\mathrm{~s}, 3 \mathrm{H}), 5.04(\mathrm{~s}, 2 \mathrm{H}), 6.69$ (d, $J=8.80 \mathrm{~Hz}, 2 \mathrm{H}), 6.99$ (d, $J=8.00 \mathrm{~Hz}, 2 \mathrm{H}), 7.22$ (s, 1H), 7.31 (d, $J=8.40 \mathrm{~Hz}, 2 \mathrm{H}), 7.48$ (d, $J=8.42 \mathrm{~Hz}, 2 \mathrm{H})$, $7.76(\mathrm{~s}, 1 \mathrm{H}) ;{ }^{13} \mathrm{C}$ NMR $\left(101 \mathrm{MHz}, \mathrm{CDCl}_{3}\right) \delta: 14.96,20.58$, 34.21 , 67.54, 99.65, 115.17, 122.47, 125.55, 126.31, $129.17,130.43,133.34,134.35,142.08,147.03,148.56$, 151.06, 154.64, 160.42. Anal. calcd for $\mathrm{C}_{23} \mathrm{H}_{21} \mathrm{ClN}_{4} \mathrm{O}_{3}: \mathrm{C}$ 63.23, H 4.85, N 12.82; found C 63.40, H 4.98, N 12.68.

5-(4-叔丁基苯氧基)-1,3-二甲基- $1 H$-吡唑-4-甲酰基$O$-\{[5-(4-氯苯基)噁唑-2-基]甲基 $\}$ 肜(9r)：黄色油状物， 产率 71\%. ${ }^{1} \mathrm{H}$ NMR (400 MHz, $\left.\mathrm{CDCl}_{3}\right) \delta: 1.29$ (s, 9H), $2.36(\mathrm{~s}, 3 \mathrm{H}), 3.60(\mathrm{~s}, 3 \mathrm{H}), 5.11(\mathrm{~s}, 2 \mathrm{H}), 6.79(\mathrm{~d}, J=8.82$ $\mathrm{Hz}, 2 \mathrm{H}), 7.29$ (d, $J=8.80 \mathrm{~Hz}, 3 \mathrm{H}), 7.38$ (d, $J=8.42 \mathrm{~Hz}$, 2H), $7.56(\mathrm{~d}, J=8.40 \mathrm{~Hz}, 2 \mathrm{H}), 7.84(\mathrm{~s}, 1 \mathrm{H}) ;{ }^{13} \mathrm{C}$ NMR $(101$ $\left.\mathrm{MHz}, \mathrm{CDCl}_{3}\right) \delta: 15.04,31.42,34.23,34.31,67.54,99.73$, $114.76,122.48,125.57,126.30,126.84,128.73,129.18$, $131.40,134.38,142.10,146.72,147.02,148.58,151.10$, 154.52, 160.39. Anal. calcd for $\mathrm{C}_{26} \mathrm{H}_{27} \mathrm{ClN}_{4} \mathrm{O}_{3}: \mathrm{C} 65.20, \mathrm{H}$ 5.68, N 11.70; found C 65.05, H 5.81, N 11.86.

5-(4-甲氧基苯氧基)-1,3-二甲基- $1 H$-吡唑-4-甲酰基$O$-\{[5-(4-氯苯基)噁唑-2-基]甲基 $\}$ 肜(9s): 黄色油状物, 产率 $82 \%$. ${ }^{1} \mathrm{H}$ NMR $\left(400 \mathrm{MHz}, \mathrm{CDCl}_{3}\right) \delta: 2.28(\mathrm{~s}, 3 \mathrm{H})$, 3.53 (s, 3H), 3.68 (s, 3H), 5.04 (s, 2H), 6.74 (s, 4H), 7.22 (s, 1H), 7.31 (d, $J=8.38 \mathrm{~Hz}, 2 \mathrm{H}), 7.49$ (d, $J=8.80 \mathrm{~Hz}$, 2H), $7.74(\mathrm{~s}, 1 \mathrm{H}) ;{ }^{13} \mathrm{C}$ NMR (101 MHz, $\left.\mathrm{CDCl}_{3}\right) \delta: 14.90$, $34.20,55.68,67.55,99.41,114.96,116.47,122.50,125.56$, 126.31, 129.17, 131.40, 134.35, 142.02, 147.03, 148.91, $150.54,151.05,155.87,160.40$. Anal. calcd for $\mathrm{C}_{23} \mathrm{H}_{21} \mathrm{Cl}-$ $\mathrm{N}_{4} \mathrm{O}_{4}$ : C 61.00, H 4.67, N 12.37; found C 61.16, H 4.55, N 
12.22.

5-(4-三氟甲氧基苯氧基)-1,3-二甲基- $1 H$-吡唑-4-甲 酰基- $O$ - $\{[5-(4-$ 氯苯基)啞唑-2-基 $]$ 甲基 $\}$ 肜(9t): 黄色固 体, 产率 75\%. m.p. $113 \sim 115{ }^{\circ} \mathrm{C}$; ${ }^{1} \mathrm{H}$ NMR $(400 \mathrm{MHz}$, $\left.\mathrm{CDCl}_{3}\right) \delta: 2.27(\mathrm{~s}, 3 \mathrm{H}), 3.53(\mathrm{~s}, 3 \mathrm{H}), 5.00(\mathrm{~s}, 2 \mathrm{H}), 6.81(\mathrm{~d}$, $J=9.18 \mathrm{~Hz}, 2 \mathrm{H}), 7.06(\mathrm{~d}, J=8.82 \mathrm{~Hz}, 2 \mathrm{H}), 7.21$ (s, 1H), 7.31 (d, $J=8.80 \mathrm{~Hz}, 2 \mathrm{H}), 7.48$ (d, $J=8.82 \mathrm{~Hz}, 2 \mathrm{H}), 7.78$ $(\mathrm{s}, 1 \mathrm{H}) ;{ }^{13} \mathrm{C} \mathrm{NMR}\left(101 \mathrm{MHz}, \mathrm{CDCl}_{3}\right) \delta: 14.59,34.28$, $67.58,99.82,116.38,119.13,122.49,122.85,125.53$, $126.27,129.18,134.40,141.56,144.81,147.28,147.46$, 151.05, 154.84, 160.24. Anal. calcd for $\mathrm{C}_{23} \mathrm{H}_{18} \mathrm{ClF}_{3} \mathrm{~N}_{4} \mathrm{O}_{4}$ : C 54.50, H 3.58, N 11.05; found C 54.32, H 3.73, N 11.19.

辅助材料(Supporting Information) 目标化合物 9a 9t 的核磁共振氢谱和碳谱图谱. 这些材料可以免费从本 刊网站(http://sioc-journal.cn/)上下载.

\section{References}

[1] Prakash, T. B.; Reddy, G. D.; Padmaja, A.; Padmavathi, V. Eur. J. Med. Chem. 2014, 82, 347.

[2] Guan, A. Y.; Qin, Y. K.; Wang, J. F.; Li, B. J. Fluorine Chem. 2013, $156,120$.

[3] Li, G. Y.; Qian, X. H.; Cui, J. N.; Huang, Q. C.; Zhang, R.; Guan, H. J. Agric. Food Chem. 2006, 54, 125.

[4] Ohnmacht, S. A.; Ciancimino, C.; Vignaroli, G.; Gunaratnam, M.; Neidle, S. Bioorg. Med. Chem. Lett. 2013, 23, 5351.

[5] Kalinin, S.; Valtari, A.; Ruponen, M.; Toropainen, E.; Kovalenko, A.; Nocentini, A.; Gureev, M.; Darin, D.; Urtti, A.; Supuran, C. T.; Krasavin, M. Bioorg. Med. Chem. 2019, 27, 115086.

[6] Wang, M. M.; Zhang, Q. Q.; Yue, K.; Li, Q. S.; Xu, F. B. Chin. J. Org. Chem. 2017, 37, 1774 (in Chinese). (王梦梦, 张青青, 岳凯, 李庆山, 徐风波, 有机化学, 2017, 37, 1774.)

[7] Mao, M. Z.; Li, Y. X.; Zhou, Y. Y.; Zhang, X. L.; Liu, Q. X.; Di, F. J.; Song, H. B.; Xiong, L. X.; Li, Y. Q.; Li, Z. M. J. Agric. Food Chem. 2014, 62, 1536.

[8] Fu, Y.; Zhang, D.; Kang, T.; Guo, Y. Y.; Chen, W. G.; Gao, S.; Ye, F. Bioorg. Med. Chem. Lett. 2019, 29, 570.
[9] Li, Y.; Zhang, H. Q.; Liu, J.; Yang, X. P.; Liu, Z. J. J. Agric. Food Chem. 2006, 54, 3636.

[10] Dai, H.; Xiao, Y. S.; Li, Z.; Xu, X. Y.; Qian, X. H. Chin. Chem. Lett. 2014, 25, 1014.

[11] Dai, H.; Ge, S. S.; Guo, J.; Chen, S.; Huang, M. L.; Yang. J. Y.; Sun, S. Y.; Ling, Y.; Shi, Y. J. Eur. J. Med. Chem. 2018, 143, 1066.

[12] Zhong, L. K.; Jiang, T.; Zhang, F.; Fu, Q.; Liu, X. H.; Xu, T. M.; Ding, C. R.; Chen, J.; Yuan, J.; Tan, C. X. Chin. J. Org. Chem. 2019, 39, 2655 (in Chinese).

(钟良坤, 江涛, 张帆, 付庆, 刘幸海, 许天明, 丁成荣, 陈杰, 袁静，谭成侠，有机化学, 2019, 39, 2655.)

[13] Park, H. J.; Lee, K.; Park, S. J.; Ahn, B.; Lee, J. C.; Cho, H. Y.; Lee, K. I. Bioorg. Med. Chem. Lett. 2005, 15, 3307.

[14] Ouyang, G. P.; Cai, X. J.; Chen, Z.; Song, B. A.; Bhadury, P. S.; Yang, S.; Jin, L. H.; Xue, W.; Hu, D. Y.; Zeng, S. J. Agric. Food Chem. 2008, 56, 10160.

[15] Dai, H.; Chen, J.; Li, G.; Ge, S. S.; Shi, Y. J.; Fang, Y.; Ling, Y. Bioorg. Med. Chem. Lett. 2017, 27, 950.

[16] Hamaguchi, H.; Kajihara, O.; Katoh, M. J. Pestic. Sci. 1995, 20, 173.

[17] Swanson, M. B.; Ivancic, W. A.; Saxena, A. M.; Allton, J. D.; O’Brien, G. K.; Suzuki, T.; Nishizawa, H.; Nokata, M. J. Agric. Food Chem. 1995, 43, 513.

[18] Fu, C. R.; Peng, J.; Ning, Y.; Liu, M.; Shan, P. C.; Liu, J.; Li, Y. Q.; Hu, F. Z.; Zhu, Y. Q.; Yang, H. Z.; Zou, X. M. Pest Manage. Sci. 2014, 70, 1207.

[19] Dai, H.; Li, G.; Chen, J.; Shi, Y. J.; Ge, S. S.; Fan, C. G.; He, H. B. Bioorg. Med. Chem. Lett. 2016, 26, 3818.

[20] Dai, H.; Chen, J.; Hong, Y.; Yuan, B. Y.; Fan, C. G.; Ma, R. Y.; Liang, Z. P.; Shi, J. Chin. J. Org. Chem. 2017, 37, 1542 (in Chinese).

(戴红, 陈佳, 洪宇, 袁斌颖, 范崇光, 马瑞媛, 梁志鹏, 石健, 有机化学, 2017, 37, 1542.)

[21] Ding, C. R.; Pan, Y. Y.; Yin, X.; Tan, C. X.; Zhang, G. F. Chin. J. Org. Chem. 2019, 39, 836 (in Chinese).

(丁成荣, 潘亚运, 殷许, 谭成侠, 张国富, 有机化学, 2019, 39, 836.)

[22] Dai, H.; Yao, W.; Fang, Y.; Sun, S. Y.; Shi, Y. J.; Chen, J.; Jiang, G. Q.; Shi, J. Molecules 2017, 22, 2000.

[23] Krasavin, M.; Korsakov, M.; Dorogov, M.; Tuccinardi, T.; Dedeoglu, N.; Supuran, C. T. Eur. J. Med. Chem. 2015, 101, 334.

[24] Park, M. S.; Park, H. J.; Park, K. H.; Lee, K. I. Synth. Commun. 2004, 34, 1541.

[25] Dai, H.; Ge, S. S.; Li, G.; Chen, J.; Shi, Y. J.; Ye, L. Y.; Ling, Y. Bioorg. Med. Chem. Lett. 2016, 26, 4504. 